\title{
artículos
}

\section{El registro del MoMo (Movimiento Moderno) en Andalucía: El caso de Andújar}

\author{
Rafael Casuso Quesada \\ Universidad de Jaén
}

RESUMEN

La ruptura con la tradición coincide en gran parte con la Segunda República y da lugar a la Arquitectura Funcionalista o Movimiento Moderno. Este novedoso proceso se corta, sólo aparentemente, con la Guerra Civil y con los principios establecidos durante la posguerra: la década de los años cuarenta y cincuenta nos ofrece un tenso equilibrio entre el ineludible progreso y la rémora de la historia, que será resuelto a favor del primero durante los años sesenta. Andalucía.

PALABRAS CLAVE: Arquitectura Contemporánea/ Movimiento Moderno/

\author{
Modern Movement (MoMo) Register in Andalusia: the example of \\ Andújar. \\ ABSTRACT \\ The break with tradition coincides mostly with the Second Republic and it provo-
} kes the Funcional Architecture or Modern Movement. This original process is broken, though only apparently, by the Civil War and the principles established during the postwar period: the forties and the fifties present a tense balance between inescapable progress and the hindiance of History, which will incline for the former one during sixties.

KEY WORDS: Contemporary Architecture/ Modern Movement/ Andalusia.

El "Movimiento Moderno", reivindicado recientemente desde las siglas MoMo, supone una auténtica revolución arquitectónica en el siglo XX, pues reinventa la idea de espacio y rompe con el decorativismo fatuo en el que había desembocado el eclecticismo decimonónico. Este movimiento se desarrolla en España a partir del año 1925 fundamentalmente, y está siendo catalogado y difundido en nuestra región desde el Instituto Andaluz de Patrimonio Histórico (IAPH), creado en 1989, y la Dirección General de Arquitectura y Vivienda, como una aportación determinante de esta centuria a la arquitectura: "El movimiento de modernización arquitectónica, difundido por todo el planeta, alcanzó su expresión plena a partir de los años veinte, se vinculó en diverso grado en España a las transformaciones políticas que tuvieron en la II República su paradigma y en el franquismo su paradoja, y se manifestó con una notable fuerza que alcanzó, en mayor o menor grado, a las principales poblaciones del territorio de Andalucía"1.

* CASUSO QUESADA, Rafael: "El registro del MoMo (Movimiento Moderno) en Andalucía: El caso de Andújar", en Boletín de Arte, n² 29, Departamento de Historia del Arte, Universidad de Málaga, 2008, págs. 375-391. 


\section{2: artículos Rafael Casuso Quesada}

En 1996 se publicó el primer registro Ibérico de Documentación y Conservación del Movimiento Moderno Ibérico (DoCoMoMo), formación más cercana del DoCoMoMo Internacional, en el que una selección de obras diseminadas por España y Portugal constituía un provisional, pero decidido elenco de referencias, con 20 ejemplos de Andalucía. La secretaría de esta organización no gubernamental recae actualmente en la fundación Mies Van der Rohe de Barcelona. La experiencia de integración de Andalucía en el DoCoMoMo, desembocó en 1997 en la elaboración por parte del Instituto Andaluz de Patrimonio Histórico, del Catálogo Andaluz de Arquitectura Moderna. Se recogían 200 edificios en este catálogo, construidos entre los años 1925 y 1965, o sea en los límites temporales que suponen el inicio del Movimiento Moderno y la fecha de creación de la primera Escuela de Arquitectura en Andalucía, la de Sevilla.

El registro citado tiene una especial importancia para Andújar, por ser una ciudad pionera en la implantación del MoMo en Andalucía: la construcción andaluza más antigua que se adscribe a este movimiento es la Presa del Jándula (Casto Fernández Shaw y Carlos Mendoza, 1924-1932). Además, incluye otras obras de la ciudad como la Viña Gisbert (José Corbella, 1932-1934), el Cine Tívoli (Fernando Alzado y José Corbella, 1933-1934). El objetivo de esta investigación no es sólo resaltar la importancia de estas edificaciones, sino ponerlas en relación con otras obras provinciales que también se incluyen en el registro: Viviendas Protegidas de Jaén (Francisco López, Julián Laguna y Juan Piqueras, 1945), Edificio de Correos de Úbeda (Alejandro de la Sota, 1964), Poblado de Miraelrío de Vilches (José Antonio Fernández del Amo, 1964) y Fábrica TECOSA de La Carolina (Fernando Higueras, 1966).

La divulgación del Movimiento Moderno ha tenido su paradigma últimamente en la exposición montada por las Consejerías de Obras Públicas y Cultura de la Junta de Andalucía, donde se exponían las 64 obras más representativas del MoMo en Andalucía. Durante la presentación de la misma por parte de uno de los comisarios de la exposición, Ramón Pico, se hizo hincapié en la necesidad de agilizar los trámites para que se pueda ampliar el registro de las 200 obras inicialmente planteado. Se propuso, más concretamente, la inclusión de tres edificios sin catalogar, pero muy representativos de su tiempo y de un notable interés patrimonial y técnico: la Plaza de Abastos de Andújar (Pedro Rivas, 1939) y la Estación de Autobuses de Jaén (Sánchez Ballesta y De la Peña, 1941), ambas con impresionantes voladizos de hormigón armado, y el Seminario de los Padres Paúles de Andújar (Luis Laorga, 1966), un desconocido complejo arquitectónico que puede resultar fundamental para entender la arquitectura en Andalucía durante la década de los sesenta.

El reconocimiento administrativo y patrimonial a esta trascendente aportación arquitectónica ha llegado en 2006, con la inclusión de la Presa del Jándula en el Catálogo General del Patrimonio Histórico Andaluz determinado por la Consejería de Cultura, junto a otras obras de Andújar, la Viña Gisbert y el Cine Tívoli, así como la

1 PÉREZ ESCOLANO, Víctor y FERNÁNDEZ-BACA CASARES, Román. "Presentación", en AA. VV: Momo
Andalucía. Arquitectura del Movimiento Moderno en Andalucía (1925-1965). Sevilla, Consejería de Obras Públicas y Transportes - Consejería de Cultura. Junta de Andalucía, 1999, pág. 7. 
Q: artículos El registro del MoMo (Movimiento Moderno)...

Estación de Autobuses y las Viviendas Protegidas de Jaén. La labor divulgativa del Movimiento Moderno está teniendo también un considerable impulso desde el Instituto Andaluz de Patrimonio Histórico ${ }^{2}$. De hecho, el Registro Andaluz de Arquitectura Contemporánea en la actualidad ya contempla las primeras treinta obras de la provincia de Jaén, entre las que se encuentran las ya citadas de Andújar pertenecientes al Movimiento Moderno: la Plaza de Abastos y el Seminario. También se introduce en este registro el Poblado de Colonización de los Llanos del Sotillo realizado entre los años 1956 y 1966 según proyecto del arquitecto José Antonio Corrales Gutiérrez.

\section{Algunas consideraciones sobre el MoMo.}

El denominado "movimiento moderno" en la arquitectura comienza en dos ámbitos geográficos muy distantes que influirán posteriormente en su desarrollo: el americano y el europeo. El primero de ellos es el más antiguo, anclado en el siglo XIX dentro de la "Escuela de Chicago", y surge sin los prejuicios antimaquinistas que estaban presentes en la arquitectura europea de entonces; a salvo, además, del encorsetamiento de la tradición, lo que le evitaba arrastrar la lacra del historicismo. Sus edificios se iban a proyectar según las exactas conveniencias del presente, aupando al rascacielos como su mejor símbolo gracias a la aplicación de la luz eléctrica y al uso de las estructuras metálicas. El utilitarismo de los arquitectos americanos, que trajo consigo la especulación del suelo al aprovechar al máximo los solares disponibles, estará en la base del denominado "organicismo". Así se observa en obras del arquitecto Luis Sullivan, como los Almacenes Carlson (1889), donde se anticipa el uso de la ventana apaisada y la idea de priorizar la funcionalidad de la obra a su estética: la belleza de un edificio radica en su propia estructura y no en ningún añadido posterior, por lo que Sullivan propone la renuncia al empleo de ornamentos en la arquitectura durante algunos años. Su discípulo, Frank LLoyd Wright consolidará el movimiento organicista y le dará su dimensión internacional, ya en el siglo XX. En sus comienzos Wright plasmó estas premisas en la proyección de casas unifamiliares urbanas, las llamadas "casas de la pradera", para la alta sociedad de Chicago; en ellas creó un modelo muy interesante a base de módulos rectangulares que se organizaban como bloques en torno a un núcleo central, donde se situaba la chimenea u "hogar" (Hickox House, 1900). Otra característica de la arquitectura orgánica que se observa en estas construcciones es la de integrar la casa en la naturaleza, dotándola al exterior de materiales constructivos tradicionales, como la piedra, la madera o el ladrillo.

La segunda corriente dentro del movimiento moderno, la denominada "racionalista", surge en Europa y de forma más tardía, debido a que la eclosión del Modernismo a principios del siglo XX contribuyó a perpetuar el mensaje de la arqui-

2 En algunas ocasiones, no obstante, se ignora la rica aportación de la provincia de Jaén: BONO RUIZ DE LA HERRÁN, Luis - GARCÍA V., Carlos - PÉREZ ESCOLANO, Víctor y PICO VALIMAÑA, Ramón: "Veinte obras del Movimiento Moderno en Andalucia", en P.H. Boletín del Instituto Andaluz del Patrimonio Histórico (Dossier DOCOMOMO), n 15, Sevilla, 1996, págs. 133-152. 


\section{Q: artículos Rafael Casuso Quesada}

tectura ecléctica. Fue dentro de la Secesión vienesa donde se dieron los principales síntomas de renovación de la arquitectura en Europa, pues los arquitectos austriacos querían distinguirse de sus colegas modernistas en Bélgica, Francia o España, reivindicando las formas geométricas elementales y eliminando cualquier atisbo de decoración. El arquitecto Otto Wagner, que también era profesor de la Academia de Viena, va a preconizar precisamente la geometrización de las líneas en sus edificios y el tejado plano, llegando a defender incluso la idea de que sólo podía ser bello lo que fuera práctico. Otro miembro de este grupo, Adolf Loos, depurará estas ideas con la publicación de un libro en 1908 de título bien sugerente, Ornamento o delito; sus conclusiones las puso en práctica en la Casa Loos (1810), erigida en Viena frente al Palacio Imperial. El modernismo, no obstante, seguía desbordando a la arquitectura europea con su derroche de imaginación.

El desarrollo de las vanguardias plásticas a principios de siglo en Europa contribuirá a la consolidación del movimiento racionalista en la arquitectura; en efecto, para los artistas modernos la pintura era, en esencia, color y la escultura, volumen. Pues bien, si algunos arquitectos ya tenían clara la necesidad de reducir al máximo la ornamentación del edificio, ahora se van a dar cuenta de que su función esencial es la de crear espacios y no la de diseñar fachadas; el exterior será una consecuencia de la articulación del espacio interior de la obra. El movimiento racionalista es profundamente individual, pero tiene algunos denominadores comunes: la simplicidad de formas, el retorno a los volúmenes elementales (cubo, cilindro, cono y esfera) y la lógica constructiva frente a la evasión ornamental. Su época dorada se desarrolla en el periodo de entreguerras, coexistiendo con la corriente organicista, y sus arquitectos más representativos son el alemán, Walter Gropius y el suizo, Le Corbusier. El primero dirige la Bauhaus desde 1919 y el segundo establece para la arquitectura un sistema de proporciones canónicas a partir de las medidas humanas (el "Modulor", 1927). Frente al organicismo, que concibe la arquitectura en función de la experiencia, modelo empírico en el que cada situación requiere una actuación independiente, la propuesta racionalista europea trata de organizar la realidad desde un plan ideal preconcebido. En Europa también surgirán en este periodo otras alternativas estilísticas como el Expresionismo (Alemania) o el Neoplasticismo (Holanda).

Los estilos modernos, por encima de la diversidad de sus propuestas, siempre coincidieron en su reacción contra el eclecticismo, un estilo que en España se perpetuaba hasta bien entrada la década de los años veinte, ahora disfrazado con ropajes regionalistas y modernistas. De ello se quejaban algunos arquitectos pertenecientes a la denominada "generación de 1925"3, como Luis Lacasa, que en el año 1926 publicaba en la revista Arquitectura, órgano de la Sociedad Central de Arquitectos, un artículo en el que afirmaba: "Es tan grande el retraso de nuestro ambiente, que tal vez no fuera demasiado optimista el pensar que hacia 1940 se intente hacer algo a la mane-

3 Denominación dada por Carlos Flores a una generación de arquitectos titulados entre los años 1918 y 1925 , cuya labor constructiva impulsó un espíritu de rebeldía contra el eclecticismo imperante, intentando incorpo- 
ra expresionista, pues no es pequeño el salto que hay que dar desde el Neomonterrey y el Neobarroco en que estamos hasta los problemas que presentan actualmente en Alemania"4. En España el mejor propagandista del nuevo estilo será el arquitecto Fernando García Mercadal, que en 1927 proyecta la que es considerada como primera obra racionalista española, el Rincón de Goya (Zaragoza). Un año más tarde es invitado a participar en el Congreso de La Sarraz (Suiza), donde la vanguardia arquitectónica europea funda el CIRPAC (Comité International pour la Réalisation des Problemes Architecturaux Contemporains), comité organizador de los futuros CIAM (Congresos Internacionales de Arquitectura Moderna).

La aceptación de estos nuevos esquemas por los arquitectos españoles iba a ser muy complicada, al menos durante la Dictadura de Primo de Rivera, y, de hecho, en 1929 se inauguran dos manifestaciones arquitectónicas de primer orden en las que vuelve a brillar con fuerza el eclecticismo: la Exposición Iberoamericana de Sevilla y la Internacional de Barcelona. La primera de ellas con un Aníbal González exultante y dispuesto a demostrar la vigencia del clasicismo en la célebre Plaza de España, y la segunda con el arquitecto Pere Domenech inundando de cúpulas barrocas el perfil de Montjuich. En la Exposición de Barcelona, no obstante, el arquitecto Mies van der Rohe iba a sorprender a todos con el prodigioso pabellón alemán, proyectado en estilo racionalista. Entre sus admiradores estaba un arquitecto recién titulado, José Luis Sert, que promoverá un movimiento renovador muy importante en el ámbito catalán, el GATCPAC (Grup d'Artistes i Tecnics Catalans per al Progrés de l'Arquitectura Contemporania) ${ }^{5}$. Este movimiento estaba influido por el racionalismo de Le Corbusier, a quien conoció Sert en 1926 durante un viaje a París y con quien entró a trabajar un año más tarde, manteniendo una intensa relación profesional a lo largo de su vida.

En 1930 el GATCPAC se iba a fundir con el grupo madrileño de la generación de 1925, creando el GATEPAC, sección española del CIRPAC, resultando ya imparable la asimilación de los ideales del movimiento moderno, que se consolidará durante la Segunda República.

Un antecedente español del MoMo: La Ciudad Universitaria DE MADRID (1927-1936).

En el mes de mayo del año 1927, en pleno auge de la dictadura del general

rar la arquitectura española al movimiento funcionalista europeo. Entre los titulados cabe citar a Luis Blanco Soler y Rafael Bergamín (1918), Casto Fernández Shaw y Miguel de los Santos (1919), Agustín Aguirre, Manuel Sánchez Arcas, Regino Borobio, José Aspiroz y Muñoz Casayús (1920), Luis Lacasa y Fernando García Mercadal (1921), Carlos Arniches, Martín Domínguez y Durán Reynals (1922) o Luis Gutierrez Soto (1923). FLORES, Carlos. Arquitectura Española Contemporánea. Bilbao, Aguilar, 1961.

4 SAMBRICIO Y RIVERA-ECHEGARAY, Carlos "Arquitectura", en Historia del Arte Hispánico. Vol. VI. El siglo $X X$. Madrid, Alhambra, 1978, pág. 30.

5 El grupo lo formaban además dos arquitectos titulados en 1925, Germá Rodríguez Arias y Francesc Fábregas, así como otro que lo fue en 1926, Ricard Churruca. ORIOL BOHIGAS. Arquitectura Española de la Segunda República. Barcelona, Tusquets, 1970, págs. 45-53. 


\section{Q: artículos Rafael Casuso Quesada}

Primo de Rivera, se publica el Real Decreto que creaba la Junta de la Ciudad Universitaria de Madrid, presidida por S.M. el rey Alfonso XIII. En un principio se pensó para su construcción en Javier de Luque, arquitecto muy afianzado durante la Dictadura y autor del ecléctico Ministerio de Educación erigido en la madrileña calle de Alcalá, pero finalmente el encargo recayó en Modesto López Otero, que tampoco era un arquitecto racionalista, si pensamos que su obra más representativa entonces era el edificio del Fénix, situado al principio de la Gran Vía madrileña. Éste facultativo, sin embargo, se rodeó de un equipo de arquitectos jóvenes, que iba a convertir la construcción de la Ciudad Universitaria en espacio de experimentación de las nuevas corrientes arquitectónicas. Era la primera vez que ello ocurría en España, al menos de una manera oficial, lo que elevó las críticas del originario proyectista, Javier de Luque:

"No, no puedo estar conforme con la arquitectura de los principales edificios de la urbe universitaria; hacen gala de rigidez, de frialdad y de extranjerismo, incompatibles con el sentir de nuestra raza y de nuestra masa estudiantil, que lleva y conserva en el alma vibraciones reñidas con esta falta de espiritualidad"6.

El impulso definitivo para la construcción del conjunto iba a llegar con la Segunda República, cuando se renovó la Junta Rectora, recayendo la presidencia en Alcalá Zamora y la secretaría en Juan Negrín; el equipo técnico fue respetado. El proyecto se truncó durante la Guerra Civil y la Ciudad Universitaria se convirtió, sin pretenderlo, en símbolo de la resistencia antifascista, al ser objeto de una cruenta destrucción en la larga batalla de Madrid. Además, algunos de sus proyectistas, como Sánchez Arcas, Rafael Bergamín y Luis Lacasa, vivieron la amarga experiencia del exilio.

El arquitecto-director del conjunto, López Otero, seleccionó a una serie de facultativos pertenecientes mayoritariamente a la "generación de 1925", entre los que se distribuyeron los distintos encargos. Siguiendo criterios urbanísticos centroeuropeos se va a aplicar una idea muy novedosa, la de agrupar los edificios docentes y residenciales para los estudiantes en un espacio común, de 320 hectáreas de terreno; en él se plantarían 30.000 árboles con la idea de construir sólo sobre un $10 \%$ del espacio adquirido. En los edificios se dio el tono general de inspiración racionalista, con grandes cuerpos de aspecto geométrico, desornamentado y alineamiento horizontal; la textura de la construcción, que se hace casi por completo de ladrillo rojo, siguiendo la tradición secular madrileña, introduce el guiño organicista. En el año 1936 se habían concluido ya un conjunto importante de edificios, entre los que destacan los siguientes: Fundación del Amo (Luis Blanco Soler y Rafael Bergamín, 1928-1930), Facultades de Filosofía y Letras y de Farmacia (Agustín Aguirre, 1932 y 1935), Facultades de Ciencias y Medicina (Miguel de los Santos, 1934-1936),

6 Ibidem, págs. 23-24. 
1: artículos El registro del MoMo (Movimiento Moderno)...

Escuela de Arquitectura (Pascual Bravo), Campos de Deportes (Javier Barroso), Hospital Clínico y Central Térmica (Manuel Sánchez Arcas, 1933-1936), Residencia de Estudiantes (Luis Lacasa, 1935-1936).

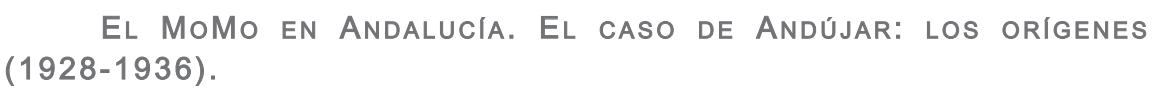

El Momo en Andalucia. El caso de Andújar: los orígenes (1928-1936).

Importante papel en la aparición del Movimiento Moderno en Jaén juegan los arquitectos Antonio Flórez Urdapilleta y Luis Berges, después de una larga trayectoria proyectiva dentro del tardo-eclecticismo, llámese regionalista o neomudejarista; un caso similar a lo que sucederá en Andujar con el constructor José Corbella. El primero de ellos lo hace desde la sabia conjugación de los volúmenes puros y la depuración ornamental con la monumentalidad del historicismo, visible en la Escuela Normal de Magisterio de Jaén (1920), que estilísticamente rememora el espíritu de la arquitectura montañesa resucitado por Leonardo Rucabado a principios de siglo. Antonio Flórez, desde la proyección de la Residencia de Estudiantes de Madrid (1911), también reivindicó el ladrillo en su dimensión auténtica y funcional, despojada de los lenguajes historicistas, especialmente el tan manido mudejarismo, y defendió también la pureza volumétrica de la arquitectura ${ }^{7}$. De ello va a dejar constancia en los numerosos grupos escolares y escuelas erigidos por España, principalmente en Madrid, aunque también en otra obra realizada en Jaén, el Colegio Público "Jesús María", si bien aquí el muro aparece revocado ${ }^{8}$.

Luis Berges y José Corbella, a diferencia de Antonio Flórez, entran en el Movimiento Moderno desde una apuesta más radical. Luis Berges comienza a proyectar obras en Jaén desde el más puro convencionalismo andaluz, pero a partir de 1923 irá presentando proyectos novedosos que no ignoran los presupuestos estéticos de la Secesión Vienesa y el Art Decó, para desembocar finalmente en el funcionalismo. El cambio se observa en obras como el Sanatorio de los Prados (1928), realizado por encargo de la Diputación Provincial, para lo que recorrió el autor una serie de sanatorios franceses, alemanes y suizos; las obras se iniciaron en 1934, fueron paralizadas en gran parte durante la guerra y se modificaron con un proyecto reformado de los arquitectos Laguna y López Rivera. El Sanatorio Antituberculoso del Neveral (1930-1934), también fue objeto de importantes ampliaciones a partir de 1950, aunque conserva en su lado occidental los pabellones originarios, de funcional diseño. Otro proyecto suyo fue la antigua Escuela de Maestría Industrial (1931), hoy "I.E.S. San Juan Bosco", en cuya fachada juega con el expresionismo de las molduras geométricas tan característico del Art Decó ${ }^{9}$. Este motivo también es recu-

7 Véase al respecto GINER DE LOS RíOS, B. Cincuenta años de arquitectura española. Madrid, Adir, 1980, págs. 50-63.

8 Recientemente se ha puesto en duda la autoría de Flórez en esta obra, atribuyéndosela mejor a Luis Berges Martínez: AUTORES VARIOS. Arquitecto Berges (1891-1939). Jaén, Tinta Blanca, 2006.

9 El Art Decó tiene su origen en la Exposición de París del año 1925 y puso de moda las geometrizaciones 


\section{Q: artículos Rafael Casuso Quesada}

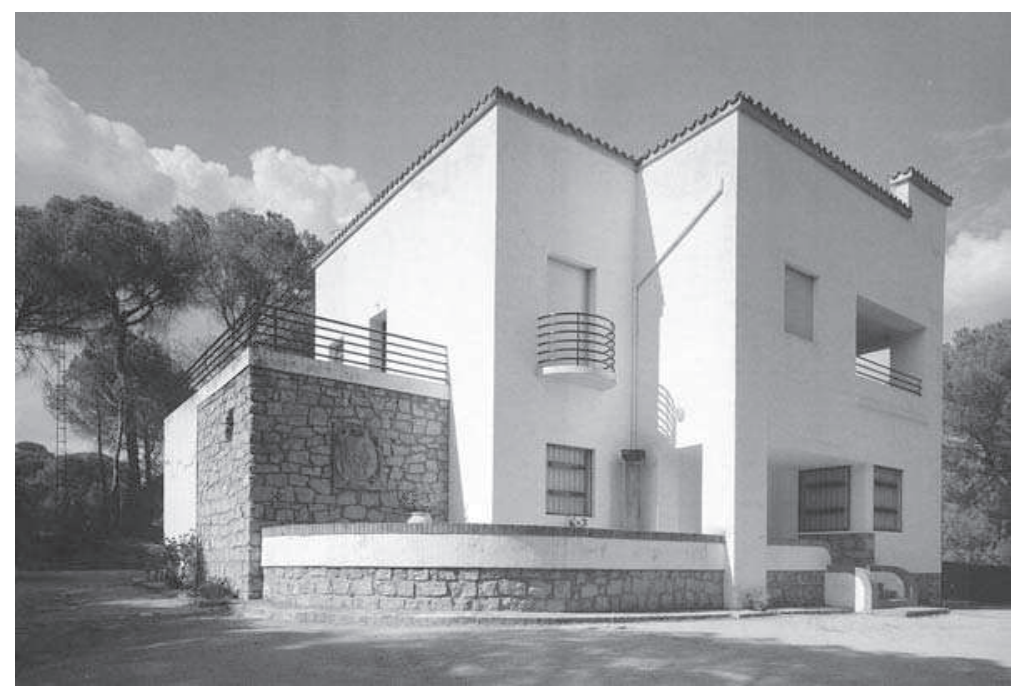

1. Viña Gisbert (1932-1934).

rrente en los Almacenes Almansa (1936), un pequeño edificio ubicado en la calle Ramón y Cajal, a espaldas de la catedral, y construido como casa particular de tres plantas, la primera de ellas para almacén. Resuelve la esquina de manera curva y juega en su diseño con bandas grises y blancas que diferencian los pisos, dentro de un racionalismo evidente. En una línea muy acorde a estos principios encontramos el Mercado de Abastos de Úbeda (Luis Casanova Vila, 1933).

El caso de José Corbella es aún más radical y ha merecido justamente la inclusión de dos de sus obras en el Catálogo Andaluz de Arquitectura Moderna: la Viña Gisbert, donde interviene como proyectista, y el CineTívoli, donde lo hace como constructor. Cuando en el año 1932 el presidente de la Junta Local de Patronales de Andújar, Plácido Gisbert, le encarga el proyecto constructivo para una casa de campo, Corbella acude a los modelos establecidos en revistas alemanas de arquitectura, a las que era muy aficionado: "No estaríamos muy descaminados al pensar concretamente en la Casa Müller (Adolf Loos, 1930), con la que el paralelismo de la Viña Gisbert es más que evidente" 10 . El edificio se caracteriza por un diseño cúbico donde

y las decoraciones cubo-futuristas de rayos y zigzags, junto con motivos naturalistas dispuestos en composiciones simétricas que explotaban las posibilidades más expresionistas del edificio: “...no sólo incluye repertorios decorativos, trae soluciones más constructivas no completamente estructurales y más bién compositivas; como son las formas escalonadas, los marcos repetidos, los arcos poligonales". PÉREZ ROJAS, J. y GARCÍA CASTELLÓN, M. El siglo XX. Persistencias y rupturas. Madrid, Silex, 1994, pág. 134. 


\section{2: artículos El registro del MoMo (Movimiento Moderno)...}

2. Salto del Jándula (1924-1932).

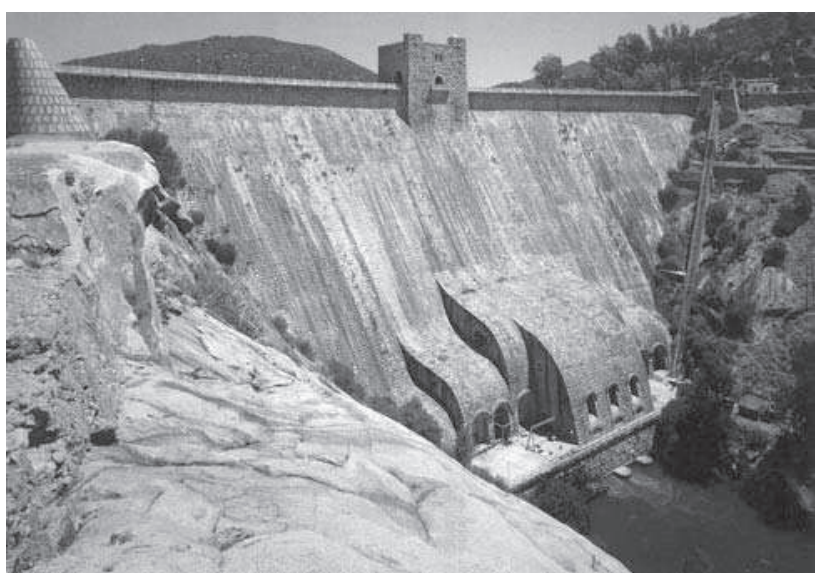

la interpenetración de volúmenes se complementa con la limpieza ornamental y el empleo de la ventana apaisada, así como con las barandillas de tubo metálico [1]. El emplazamiento de la viña en plena Sierra Morena no resulta casual, pues en esa época se estaba concluyendo una de las obras más emblemáticas del movimiento moderno en Andalucía, el Embalse del Jándula (Casto Fernández Shaw y Carlos Mendoza, 1924-1932), donde se experimentan nuevas formas de ingeniería arquitectónica, con una simbiosis formal y conceptual entre expresionismo y funcionalismo:

"el resultado funde monumentalidad estática y expresión dinámica, las connotaciones monumentales nos refieren al dominio del hombre sobre lo natural mientras que las ondulantes formas que rompen su basamento constituyen un elocuente símil de la recuperación de la libertad de las aguas contenidas"11.

Sin embargo, el poblado construido junto al pantano erigía en su parte central una hermosa ermita neorrománica, muestra inequívoca de la persistencia de valores tradicionales en la arquitectura a principios de la Segunda República [2].

En el núcleo urbano de Andújar se va a completar un episodio importante en la irrupción de la modernidad arquitectónica en Andalucía; José Corbella va a ser el hilo conductor del proceso. Entre sus intervenciones más destacadas hay que citar

10 CASUSO QUESADA, Rafael "Propuestas para una revisión crítica de la arquitectura del siglo XX en Jaén", en AA.VV. Homenaje a Luis Coronas. Jaén, Universidad de Jaén, 2001, pág. 99.

11 MORENO PÉREZ, J.R. - MOSQUERA ADELL, E. - PÉREZ CANO Mª T. "Los inicios, 1925-1936: una vanguardia de autor", En AA.VV. Momo Andalucía. Arquitectura del Movimiento Moderno en Andalucía (1925-1965). Sevilla, Consejería de Obras Públicas y Transportes - Consejería de Cultura. Junta de Andalucía, 1999, pág. 24. 


\section{Q: artículos Rafael Casuso Quesada}

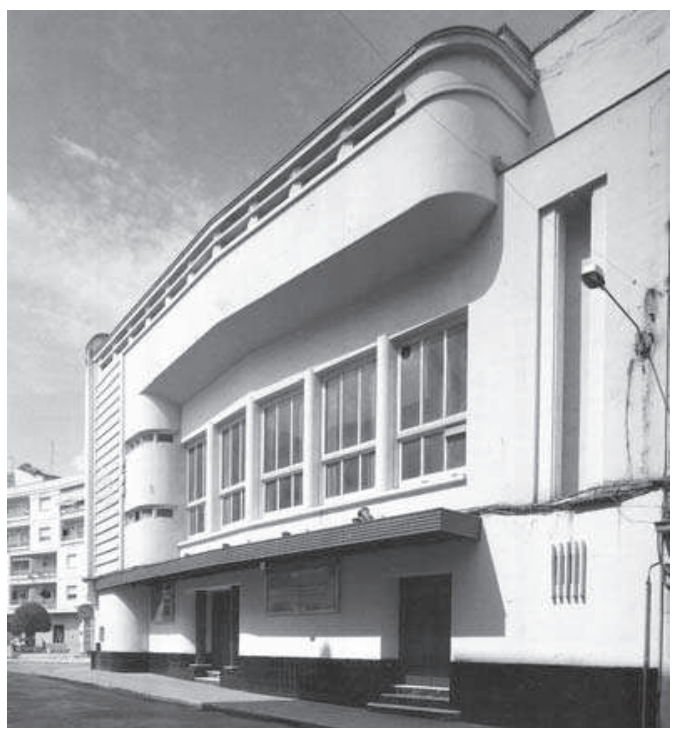

3. Cine Tívoli (1933-1934).

la planificación del solar del antiguo Castillo de Andújar, derribado en 1933 y ubicado en la Plaza Vieja, que pretende ser recuperado como un lugar aglutinador de la vida de la ciudad: un espacio multitudinario (el Cine Tívoli), una casa de pisos (la Casa Lara) y el local del Casino lliturgitano [3]. La idea que anima el proyecto es bastante coherente, pero no así resultó su definitiva resolución debido a la ausencia de un plan de diseño conjunto de los edificios. El Cine Tívoli fue proyectado en 1934 por el arquitecto madrileño Fernando Alzado, ejerciendo José Corbella un papel de dirección de la obra, mientras que en la Casa Lara sí ejerció funciones de proyectista. Mientras que el cine nos ofrece un derroche de modernidad en el empleo de los nuevos materiales, como el hormigón armado dentro de un conjunto expresionista, la Casa Lara abunda más en las propuestas de García Mercadal, en la búsqueda de una arquitectura mediterránea, de una plástica pura, limpia, horizontal, desornamentada y racionalista. José Corbella volverá a incidir en este camino en otra obra emblemática de la ciudad de Andújar, la Casa Valdés (1934), ligada además a una intervención urbana significativa:

"Una de las carencias más significativas de nuestro panorama arquitectónico funcionalista es la preocupación por la problemática urbana, aquella que, junto con el diseño moderno de las construcciones, constituyó la base más radical de la renovación social que propugnaban los arquitectos modernos" 12 .

12 CASUSO QUESADA, Rafael. Arquitectura contemporánea en Andujar (1920-1950). Andújar, Ayuntamiento y Casa de Cultura, 1990, págs. 105-106. 
1. artículos El registro del MoMo (Movimiento Moderno)...

El MoMo en la arquitectura de posguerra (1936-1965).

El exilio de Sert, Lacasa, Candela y de otros arquitectos republicanos es sintomático de la dramática experiencia vivida por toda una generación de intelectuales españoles, pero no supuso una ruptura radical con la gradual asimilación de los principios arquitectónicos del movimiento moderno, al menos desde el punto de vista estilístico. Esta cuestión, debatida polémicamente durante mucho tiempo por la historiografía antifranquista, pareció quedar más clara a raíz de la exposición Arquitectura para después de una guerra (1939-1949) que organizó el Colegio de Arquitectos de Cataluña y Baleares en Barcelona (1977), después presentada en el Museo de Arte Moderno de Madrid. En palabras de Antón Capitel, "la crítica moderna posterior a esta década pasó sobre el historicismo como si caminara sobre ascuas, la encerró en un paréntesis que nunca debería ser abierto y tendió un puente entre la arquitectura anterior a la guerra y aquella otra que, a partir de los años cincuenta, podía, por el empleo de una figuratividad moderna, establecer un tal enlace"13.

El régimen franquista, en efecto, puso en marcha toda una maquinaria administrativa con la intención de controlar la actividad urbanística y arquitectónica en España, como el Servicio de Regiones Devastadas (1938), convertido un año más tarde en Dirección General, o la Dirección General de Arquitectura, creada en 1939 y dirigida por Pedro Muguruza. También estableció un sistema de propaganda ideológica por medio de la Revista Nacional de Arquitectura, fundada en 1941 y en cuyo primer número se iba a dedicar especial interés a la obra de reconstrucción del Santuario de la Virgen de la Cabeza en Andújar (Francisco Prieto Moreno, 1941), a la que se refería de esta manera José Moreno Torres: "En la Dirección General de Regiones Devastadas, que tengo el honor de regentar, he entendido siempre que su misión no era estrictamente la de construir con fiel exactitud lo que antes existía, sino que era necesario aplicar a la reconstrucción del suelo español el sentido revolucionario del movimiento Nacional con la misma intensidad y eficacia con que se produjeron las fuerzas armadas para ganar la guerra, y así, paralelamente, estar en condiciones de ganar la paz"14. Posteriormente llegarían las medidas de depuración ideológica contra los arquitectos republicanos citadas en el epígrafe anterior, todos ellos mayoritariamente racionalistas, con lo que las orientaciones estilísticas oficiales se inclinaban hacia el respeto a la tradición constructiva y a la recuperación de los lenguajes históricos. Obras como el faraónico Valle de los Caídos (Pedro Muguruza, 1939), construido con el trabajo de presos republicanos, atestiguan el cambio.

La práctica constructiva puesta en marcha a lo largo de la década de los cuarenta contradice, sin embargo, estas pretensiones oficiales y así se observa en

\footnotetext{
13 CAPITEL, Antón “Arquitectura Española. 1939-1992”, en Arquitectura Española del siglo XX. Colección Summa Artis, vol. XL. Madrid, Espasa-Calpe, 1995, pág. 358.

14 MORENO TORRES, José "Aspectos de la reconstrucción. El Santuario de Nuestra Señora de la Cabeza", en Revista Nacional de Arquitectura. Año 1, n 1. Madrid, 1941, pág. s/n.
} 


\section{Q: artículos Rafael Casuso Quesada}

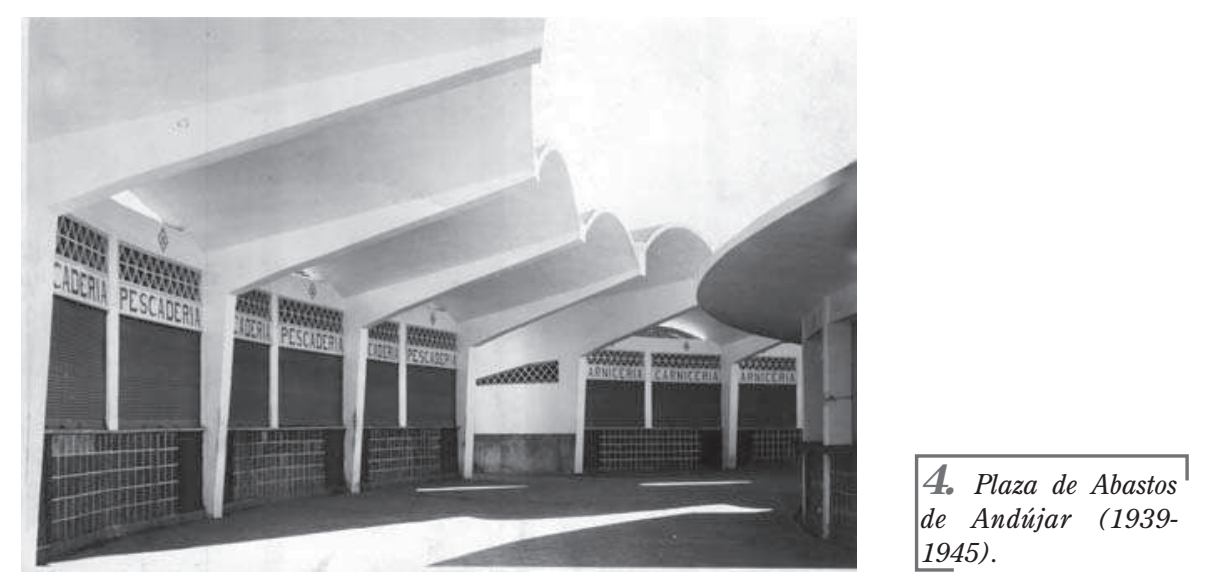

casos significativos, como el de Luis Gutiérrez Soto. Este arquitecto, miembro de la generación de 1925, recibió el encargo para la realización del Ministerio del Aire de Madrid en el año 1941, desplazándose a Italia y Alemania para imbuirse del espíritu de la arquitectura de corte fascista y presentando un primer anteproyecto inspirado en el clasicismo abstracto propio de la arquitectura oficial de estos regímenes. El "revolucionario" proyecto fue rechazado por las autoridades españolas que lo obligaron a recrear de una manera forzada la arquitectura de El Escorial, pero al mismo tiempo Gutiérrez Soto proyectaba viviendas por la capital de España al estilo de las que hiciera durante la Segunda República; estos proyectos se publicaron además, en la Revista Nacional de Arquitectura en el año 1943:

"En la posguerra, a pesar de los alardes verbales, no se puede hablar de un estilo único, sólidamente sistematizado, plataforma para la crítica histórica de la arquitectura y para la constitución de un nuevo quehacer constructivo. Tampoco se pueden reconstruir los términos de un debate estilístico, que, en verdad, ni se llegó a producir. Si es cierto que, más o menos, los arquitectos falangistas entendían -y tampoco hay aquí excepciones- que la arquitectura moderna había sido manipulada, en sus aspectos semántico e ideológico... Pero todo esto no supuso una repulsa radical hacia la arquitectura moderna entre otras razones porque no había otra alternativa que no fuera la vuelta a Herrera o a Villanueva y porque en la Italia de Mussolini, la semántica ortodoxa, no era otra que el moderno racionalismo"15.

15 UREÑA PORTERO, Gabriel Arquitectura y urbanistica civil y militar en el período de la Autarquía (19361945), Madrid, Istmo, 1979, págs. 115 y 116. 


\section{Q: artículos $\quad$ El registro del MoMo (Movimiento Moderno)...}

5. Estación de Autobuses de Jaén (1920-1947).

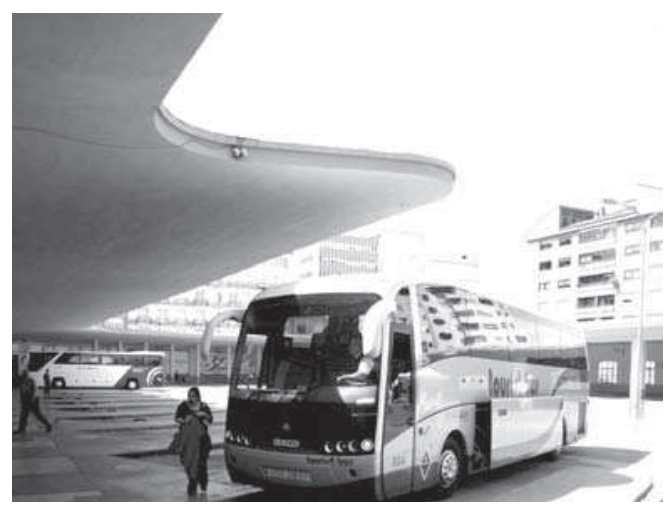

La fisura existente entre la arquitectura oficial y la promovida por particulares o entidades privadas era cada vez más evidente. En la provincia de Jaén encontramos dos proyectos muy significativos al respecto, aunque no están incluidos en el registro del DoCoMoMo:

1.- La Plaza de Abastos de Andújar (Pedro Rivas y Antonio Amat, 1939-1945). Esta obra fue promovida por la familia Rivas Sabater, concesionaria del servicio de abastos en la ciudad, tras el estado en que quedó la antigua plaza, como consecuencia de los bombardeos causados por la aviación nacional. El nuevo proyecto consta de algunos elementos de gran modernidad, como el atrevido voladizo de hormigón existente sobre el perímetro circular de la planta [4]. Éste fue diseñado por Pedro Rivas en 1939, inspirándose en aquel que hiciera el ingeniero Eduardo Torroja para el Hipódromo de la Zarzuela de Madrid (1934), en colaboración con los arquitectos Carlos Arniches y Martín Domínguez. En 1945, concluido el voladizo, se inició la construcción del cuerpo central en cuya proyección intervino el ingeniero Antonio Amat, incidiendo en el racionalismo del conjunto. Sólo un juicio apriorístico consideraría incompatible la modernidad de esta obra con el pensamiento de un arquitecto franquista como Pedro Rivas, que firmó el proyecto en Madrid con el saludo "Año de la Victoria“.

2.- La Estación de Autobuses de Jaén (1940-1947). Promovida por la compañía Auto Estaciones S.A., en ella destaca una sorprendente marquesina de hormigón que los arquitectos Sánchez Ballesta y De La Peña proponen en el proyecto de 1941 [5]; una muestra más del enlace con los estudios de resistencia de materiales que iniciara Torroja durante la Segunda República. La obra, además, "se va a constituir en imprescindible punto de referencia para las nuevas generaciones de arquitectos que desarrollarán su labor en la ciudad en años sucesivos"16. En comparación con otros

16 CASUSO QUESADA, Rafael “La Estación de Autobuses de Jaén: una importante aportación a la arqui- 
edificios de semejante funcionalidad, como la Estación de Autobuses de Almería (Guillermo Langle, 1952), el edificio de Jaén implica una apuesta técnica de mayor riesgo 17.

La extremada matización con la que se aplicaban en arquitectura las proclamas oficiales iba aumentando conforme se alejaba en el tiempo el recuerdo de la guerra, incluso en edificios tan representativos del régimen como la Delegación Nacional de Sindicatos en el Paseo del Prado de Madrid. En el año 1948 esta obra fue objeto de un concurso de proyectos del que resultó ganador el presentado por Francisco Cabrero y Rafael de Aburto, un rascacielos de gran lógica constructiva situado en las antípodas de la retórica imperial. El edificio resuelve el problema de la escala retranqueando el bloque en altura para confiar al cuerpo bajo la mediación con los edificios preexistentes. Quedaba evidenciada, casi a final de la década, la derrota de los postulados arquitectónicos franquistas, ante los presupuestos de la arquitectura racionalista, más arraigados entre nuestros facultativos de lo que pensaban los ideólogos del nuevo régimen.

De esta época el registro del MoMo sólo recoge el conjunto de las Viviendas Protegidas de Jaén (Francisco López, Julián Laguna y Juan Piqueras, 1945), de pobre diseño, por su condición de viviendas sociales, pero de inteligente articulación urbanística:

\begin{abstract}
"agrupándose en grandes manzanas de 125, 136 y 150 viviendas, respectivamente, que siguen una estrategia de ocupación del solar claramente influida por los modelos centroeuropeos de vivienda social, al protegerse de una escasamente atractiva periferia para volcarse hacia un cuidado espacio interior ocupado por grandes patios que se conectan mediante recorridos transversales de acceso rodado" 18 .
\end{abstract}

Sin adquirir esta dimensión, en Andújar vamos a encontrar ejemplos significativos de viviendas protegidas: “...aquellas que, acogiéndose a las ventajosas condiciones establecidas en el decreto-ley del 25 de Noviembre de 1948, van a dinamizar la labor constructiva y permitirán a muchos ciudadanos acceder a la compra o alquiler de una casa por primera vez"19. Éstas se van a distribuir por la zona norte de la ciudad y nacen como complemento funcional del centro urbano, reforzado considerablemente, y dependientes del mismo. La más antigua es la del Barrio del Matadero

tectura española de posguerra", en Revista Senda de los Huertos, $n^{\circ}$ 41. Jaén, Asociación de Amigos de San Antón, 1996, pág. 59.

17 Recientemente se ha editado una monografía sobre el edificio anticipándose a su inclusión en el Catálogo General del Patrimonio Histórico Andaluz, que se produjo en el año 2006: QUESADA GARCíA, Santiago y CASUSO QUESADA, Rafael. La estación de autobuses de Jaén. Sevilla, Consejería de Cultura de la Junta de Andalucía, 2005

18 BENITEZ, Jorge y ASENSIO, José Miguel “Inmigraciones, 1950-1965: periferias, polígonos y viviendas sociales", en AA.VV. Momo Andalucía. Arquitectura del Movimiento Moderno en Andalucía (1925-1965). Sevilla, Consejería de Obras Públicas y Transportes - Consejería de Cultura. Junta de Andalucía, 1999, pág. 210.

19 CASUSO QUESADA, Rafael "Evolución y cambio en la arquitectura contemporánea de Andujar", en Boletín del Instituto de Estudios Giennenses, n 181. Jaén, Instituto de Estudios Giennenses, 2002, pág. 207. 
(Pedro Rivas, 1949), aunque destaca especialmente la urbanización de los alrededores de la Plaza de Toros, alternándose viviendas unifamiliares con bloques de pisos. Su norma estilística va a ser la sencillez, para evitar costes, y un toque estético ruralizante.

El catálogo andaluz del MoMo pasa de puntillas por los años cincuenta, pues no inscribe ningún edificio de la provincia. En el caso de Andújar no faltarían motivos para afirmar que en esa década se vive una edad dorada de la construcción privada, pues van a proyectar edificios para la ciudad arquitectos tan renombrados como Carlos Arniches y Martín Domínguez (antiguo Hotel don Pedro, recogido en el Registro Andaluz de Arquitectura Contemporánea), Rafael de la Hoz, José María García de Paredes, Felipe López Delgado, José Fernández del Amo o Pedro Bigador, aunque la mayoría desde una perspectiva neohistoricista, alejada de los presupuestos estéticos del Movimiento Moderno20. En cualquier caso justo es reivindicarla desde estas páginas, ya que algún que otro edificio de este conjunto ha sido ya derribado; nos referimos concretamente a la casa de la calle 22 de Julio $n^{\circ} 16$, frente al convento de las Trinitarias, promovida por Damián Parras y diseñada por los arquitectos Rafael de la Hoz y José Ma García de Paredes.

Los años sesenta suponen una renovada eclosión del funcionalismo, una renovación estilística y estructural que se va a iniciar en obras ligadas al "boom turístico" como el Camping (Ramón Pajares, 1964), o el Hotel Del Val (Mateos y Ontañón, 1962). Desgraciadamente, la primera de ellas ya ha pasado a engrosar la historia de los recientes "desaguisados" contra edificios emblemáticos de la arquitectura contemporánea iliturgitana, como el del Cine Avenida; estos derribos, además, han afectado a la limitación de servicios públicos de primer orden, paradójicamente desde la perspectiva de un Plan General de Ordenación Urbana, el de 1989, de pretendido carácter progresista:

"Entre los aspectos del plan que han resultado más negativos cabe citar la actuación en el antiguo Camping, con un volumen de superficie construida que ha privado a Andujar de un elemento de servicio y un pulmón verde fundamentales. Asimismo, la realización de un gran espacio escénico en la zona sigue siendo una asignatura pendiente, evidenciándose lo absurdo del derribo del Cine Avenida y su transformación en viviendas particulares"21.

De esta década el Catálogo Andaluz del Movimiento Moderno no selecciona ningún edificio de Andújar, aunque sí lo hace con otros tres significativos de la provincia de Jaén: Edificio de Correos de Úbeda (Alejandro de la Sota, 1964), Poblado de

20 Recientemente ha sido reivindicada esta época por PERAL LÓPEZ, José "Arquitectura contemporánea de Andujar. Mejor sumar que restar. Años 40, 50 y 60". Comunicación leída en II.Curso de Historia, Arte y Cultura de Andujar (Universidad de Otoño, 2002). En prensa.

21 CASUSO QUESADA, Rafael Antonio "Evolución urbanística de Andujar en el siglo XX", en AA.VV. Anuario de Investigaciones Hésperides - Alcalá la Real. Sevilla, Asociación Hespérides, 2001. pág. 76. 


\section{Q: artículos Rafael Casuso Quesada}

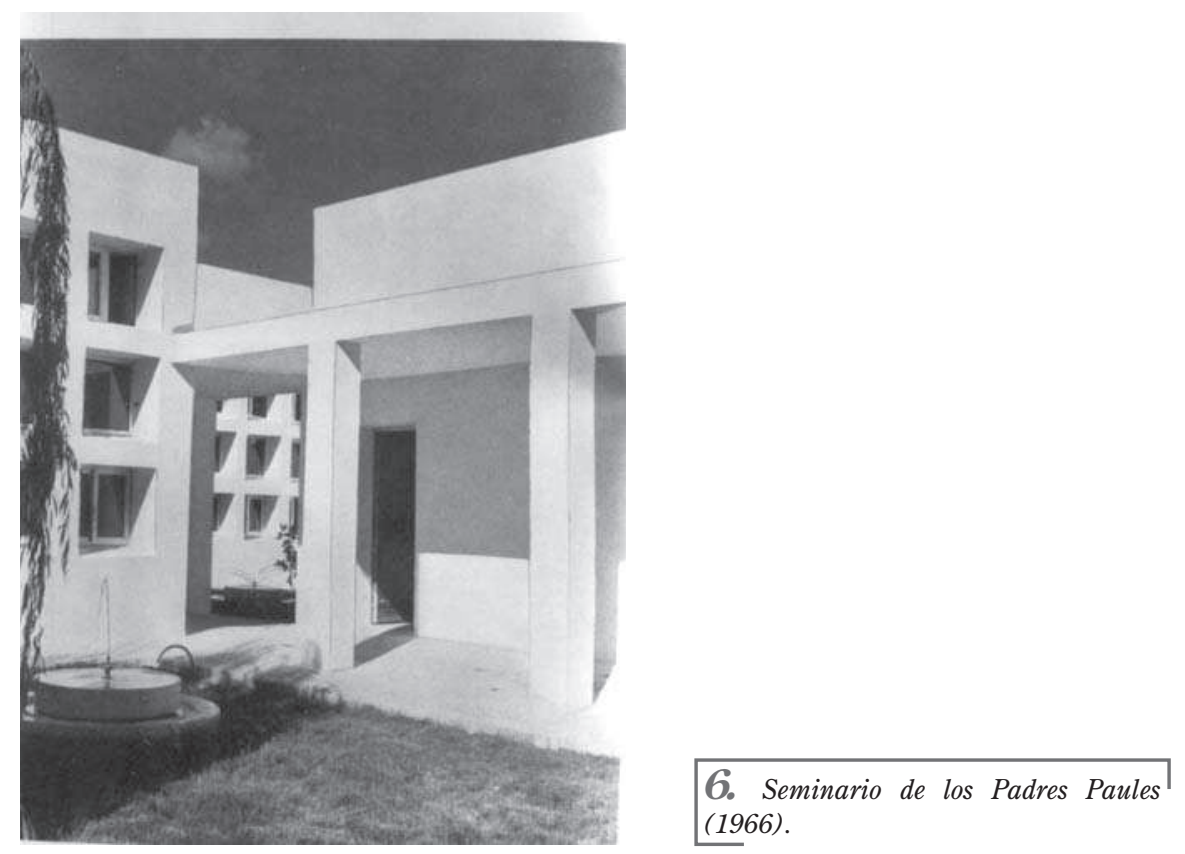

Miraelrío de Vilches (José Antonio Fernández del Amo, 1964) y Fábrica TECOSA de La Carolina (Fernando Higueras, 1966). Al primero se le inscribe como ejemplo de intervención inteligente en un casco histórico artístico consolidado, sin renunciar a su diseño moderno y dialogando activamente con un convento y un palacio a cada uno de sus laterales. Del poblado de Miraelrío en Vilches destaca no tanto su diseño de fachadas, como la articulación del espacio a lo largo de un amplio círculo donde se disponen las viviendas unifamiliares, las cuales giran en torno a un centro de espacios y dependencias comunes. Aunque pendientes de un estudio pormenorizado, los poblados de colonización del entorno geográfico de Andújar también nos ofrecen soluciones dignas de tener en cuenta, especialmente en lo que se refiere al Poblado de los Llanos del Sotillo, proyectado por José Antonio Corrales en 1956 e inscrito en el Registro Andaluz de Arquitectura Contemporánea, anterior en el tiempo al poblado de Vilches y con una iglesia de depurado diseño moderno. De la Fábrica Tecosa de La Carolina cabe destacar la magnífica articulación del espacio e iluminación interior con el empleo de paraboloides hiperbólicos de hormigón como soportes. Ejemplo del despegue económico e industrial de los años sesenta, también tenemos en Andújar una réplica a tener en cuenta, el Complejo Industrial de Koipe, aunque en este caso el diseño de las construcciones obedece a otros criterios menos técnicos.

Consideramos, en cualquier caso, imprescindible la inclusión en el catálogo 


\section{2: artículos El registro del MoMo (Movimiento Moderno)...}

del Seminario de los PP. Paules (1966), uno de los edificios mas emblemáticos de los aquí estudiados, incluso a nivel nacional. Fue proyectado por el arquitecto Luis Laorga, el mismo que construyera unos años antes el Santuario de la Virgen de Aránzazu, auténtico manifiesto de la vanguardia artística española de la época. La obra se va a caracterizar por su atrevido diseño exterior, de marcado carácter expresionista, y de una planta en forma angular en la que el presbiterio se sitúa en la esquina que resulta de la unión de dos naves; una verdadera novedad dentro de la tradición arquitectónica religiosa ${ }^{22}$. La funcionalidad del Seminario no se debe tanto a principios estilísticos como a una cuestión de perentoriedad económica, que era evidente en aquellos momentos dentro de la Congregación. De este modo, el empleo de la piedra granítica iliturgitana en la decoración de los altares, introduce ahorro presupuestario, pero también un enlace con el entorno plenamente moderno [6]. Tan importante como la obra arquitectónica resulta el carácter de sus complementos en los que intervino el artista asturiano Joaquín Ruiz Carmín. Éste decoró la iglesia con vidrieras de diseño abstracto y diversos paneles cerámicos entre los que destaca un vía crucis neofigurativo y una decoración simbólica en el altar mayor alusiva a los astros y cuerpos celestes que giran en torno a la luz central. El citado artista también es autor de una serie de esculturas en hierro, destacando el Cristo central, recuerdo de Pablo Gargallo, o la Virgen, inspirada en figuras como la Montserrat de Julio González; así como de diversos útiles de uso litúrgico como custodias, palmatorias, etc...

22 ESTEBAN MOLINOS, José Arquitectura religiosa en la provincia de Jaén desde 1940 a 1971. Jaén, Instituto de Estudios Giennenses, 1982, págs. 43 y 44. 
